\title{
Un catalogue d'idées reçues sur le cancer
}

\author{
M.-F. Bacqué \\ (C) Springer-Verlag 2009
}

Les idées reçues sur le cancer foisonnent depuis l'identification de la maladie, il y a environ 2500 ans. Elles suivent l'évolution de la médecine et des sciences, mais curieusement se maintiennent dans l'imaginaire collectif [1]. Ainsi, voisinent toujours, parmi les attributions causales du cancer les plus fréquentes, celles de la faute, de la dette à payer et celle du traumatisme générant la maladie.

On observe cependant un changement d'échelle au fil des siècles : alors qu'il y a deux millénaires, la déclaration d'un cancer était perçue comme résultant d'un déséquilibre de la place de l'homme dans le cosmos, elle a suivi les moyens d'observation de l'infiniment petit. Ainsi, si les astres pouvaient être considérés comme déterminants pour la santé psychique et physique aux débuts de notre ère, le cancer a bientôt vu son origine découler d'un comportement individuel défaillant, puis, avec la découverte au microscope d'un dysfonctionnement cellulaire, et aujourd'hui enfin, d'une mutation génétique.

Discuter ici de l'objectivité de ces étiologies n'est pas le propos, ce qui est en revanche notre but est de considérer la place de ces théories (fondées ou pas) dans les représentations collectives du développement d'un cancer.

L'ensemble de ces idées persiste en effet dans l'imaginaire collectif.

\section{Représentations collectives causales du cancer}

\section{Le cancer vient d'ailleurs}

L'étiologie exogène du cancer s'est modifiée par rapport à celle qui circule toujours dans les sociétés peu industrialisées. Ce n'est plus le mauvais sort jeté qui vient posséder l'âme du malade et qui impose l'expertise d'un exorciste pour s'en débarrasser. Mais dorénavant, ce sont les événements malheureux de la vie qui se soldent par une dépression masquée ou manquée, en tout cas mal digérée, aboutissant bientôt à un cancer. On remarquera que, dans l'imaginaire collectif, il n'y a de cancer qu'au singulier.

M.-F. Bacqué $(\varangle)$

Département de psychologie, université Louis-Pasteur,

12, rue Goethe, F-67000 Strasbourg, France

e-mail :mfbacque@club-internet.fr
Comme si tous les cancers relevaient du même processus, de la même étiologie et de thérapeutiques comparables.

Bien que combattues par de nombreuses recherches alléguant l'absence de lien direct entre deuil, traumatisme et cancer, la plupart des malades et des soignants restent convaincus de cette possibilité (voir l'article de SoumPouyalet et al. dans ce numéro, qui retrouvent dans le discours de soignants et de médecins cette idée d'une psychogenèse).

Sur le plan épidémiologique, seule la corrélation dépression-âge a pu mettre en évidence une augmentation du risque de cancer. La question des effets de la dépression sur les défenses immunitaires n'étant pas encore strictement tranchée.

\section{Le cancer vient de l'intérieur}

On retrouve ici les deux niveaux de la conception endogène du cancer :

- le niveau judéo-chrétien de la dette à payer : il s'agit de la représentation classique du « coût » de la désobéissance aux contraintes sociales. Le mauvais comportement se traduit par un prix, c'est celui du cancer.

Deux conséquences : une forme de contrôle et de défiance face à des règles imposées. L'homme est un mauvais élève de la vie en société : lorsqu'il refuse de s'y plier, il en paye le prix. L'attitude qui consiste à choisir la « liberté » se doublerait également du bénéfice de ne pas vieillir, puisque grâce au cancer, le sujet ne connaîtrait pas la déchéance du grand âge ;

- le niveau beaucoup plus inquiétant de «l'alien» en soi : cette idée, tirée à la fois de l'unheimlichkeit freudienne (l'inquiétante étrangeté de ce corps familier qui nous échappe) et du scénario du film Alien de Ridley Scott (insertion, dans un individu, d'une forme inconnue de parasite dormant qui émerge de façon brutale et totalement inopinée en détruisant son hôte) est une version « moderne » de l'endogenèse du cancer.

Comment ce cancer se développe-t-il à notre insu ?

Les théories cellulaires se bousculent ici :

- Freud toujours, dans Métapsychologie, [2] avait qualifié de «narcissiques » les cellules cancéreuses. Elles se multipliaient pour leur propre profit, délaissant l'avenir commun 
de l'organisme auquel elles avaient «oublié » qu'elles appartenaient. On percevait ici, chez l'admirateur de Charles Darwin, une autre idée, celle d'une compétition nocive entre les lignées cellulaires, comme si les cellules cancéreuses, développant pour leur propre compte d'étranges qualités (perte de l'inhibition de contact, immortalité, migration, multiplication incontrôlée), détruisaient patiemment leur hôte et elles-mêmes à cette occasion ;

- les cellules cancéreuses ne connaissent pas l'apoptose. Elles diffèrent fondamentalement (et de manière contreintuitive) des autres cellules de l'organisme : leur programme génétique ne comprend pas cette forme d'autodestruction qui permet aux cellules normales " usées » de disparaître sans transmettre à leurs filles les erreurs de transcription de leur ADN.

\section{Représentations collectives thérapeutiques du cancer}

Comme François Laplantine l'avait démontré, dans son Anthropologie de la maladie en 1986, aux représentations étiologiques correspondent des conceptions thérapeutiques du cancer.

$\mathrm{Si}$ le cancer découle d'une conjoncture extérieure à l'individu, des thérapeutiques totalement étrangères le guériront.

C'est le cas des traitements comme la chirurgie qui extraie le « malin », la radiothérapie qui bombarde avec ses rayons ou encore la chimiothérapie avec ses substances éradicatrices qui circulent dans l'ensemble des vaisseaux.

Plusieurs «petits arrangements » sont alors conçus par les malades.

\section{Marchandage avec les traitements}

Un bon comportement intercèdera en faveur de la guérison. Cette position répond à l'idée de la faute. Elle pose paradoxalement des problèmes mis en évidence dans l'article de ce numéro de Regnier-Dubois et al. sur la compliance variable des patients à la chimiothérapie orale. Dans cette étude, la grande crainte des médecins n'est pas tant l'oubli de la prise des médicaments qu'au contraire une surobservance. Les patients, une fois chez eux, auraient tendance à se surtraiter, plutôt que l'inverse. Malgré les effets secondaires des traitements, ils préféreraient augmenter les doses afin d'être bien sûrs d'éliminer le cancer. De plus, ils se plaindraient beaucoup moins des conséquences des traitements : perte de poids, mucite, douleurs et même syndrome mains-pieds seraient beaucoup moins signalés du fait qu'ils feraient, tout simplement partie du prix à payer pour éliminer définitivement le cancer.

Ces questions sont d'autant plus importantes à aborder dans la prise en charge du patient atteint de cancer qu'elles jouent un rôle fondamental pour les équipes comme pour les patients : des deux côtés, elles expliquent les réticences pour la mise en place de soins à domicile.

\section{Maladie et traitements forment une épreuve initiatique}

L'idée que plus le traitement est difficile et plus il est efficace, perdure chez les malades et les médecins dans une autre optique. Ici intervient l'autre théorie thérapeutique. Il s'agit de la théorie adorcistique de la maladie comme épreuve initiatique. Dans la société occidentale moderne, la diminution des rites de passage ne donne plus le sentiment au sujet de changer de groupe d'appartenance. Beaucoup ont l'impression de «subir » une vie caractérisée par sa linéarité et un déterminisme pesant. La maladie apparât alors comme une épreuve qui pourrait, malgré un risque important, amener le sujet à modifier son mode de vie et à trouver une nouvelle position spirituelle dans l'univers. Cette théorie est actuellement la plus en vogue : on ne la retrouve pas seulement chez celles et ceux qui ont eu un cancer, mais elle peut être généralisée par tous ceux qui ont vécu une épreuve qui bouleverse une vie conçue comme préformatée. Le vocabulaire qui décrit le cancer comme une épreuve est similaire à celui qui décrivait les passages obligés des sociétés ritualisées de jadis. Les hommes et les femmes, sommés de souscrire aux lois sociales du mariage, du métier à transmettre de père en fils, des interdits, se heurtent désormais à la maladie comme une des contraintes majeures de l'existence. Cette contrainte les pousse à effectuer le «travail» spirituel qu'ils auraient accompli dans un cadre plus traditionnel et surtout plus socialisé, s'ils avaient pu intégrer un des rites de transition classique. En dehors des groupes de malades, la démarche spirituelle est principalement réalisée dans la solitude.

\section{Liens entre le « moral » et la bonne adaptation à la maladie}

Avoir «bon moral : : une «zone grise », indéterminée, entre le bien et le mal, selon Primo Levi et reprise par Soum-Pouyalet et al. pour qualifier cette entité qu'est le « moral ». Si l'on s'apprête à croire qu'il s'agit de garder l'espoir pour continuer à se soigner, une certaine déception nous gagne à la lecture de cette étude. En fait, le malade qui a bon moral est celui qui est « confortable », qui ne fait pas de vague. La notion de «bon moral » est surtout une idée de soignants. Elle est à l'origine du clivage habituel entre le corps dont ils s'occupent et la tête, qui serait confiée aux « Psy ». La séparation du moral et du reste permet d'ailleurs d'éviter le mot le plus dangereux, le fameux $D$-word (death) des Anglo-Saxons, le mot mort, chassé à grand renfort de technologie, de progrès médicaux brandis comme autant de dérisoires statistiques. Les échecs des 
traitements, lorsqu'ils ne sont pas repris par les soignants dans un travail de supervision de groupe, peuvent s'accumuler comme autant de culpabilité ou de « souffrance au travail ». Pour d'autres plus chanceux, mais tout aussi discrets, ils forment le «curriculum caché » (hidden curriculum), cette expérience existentielle acquise « sur le tas », qui permet de tirer une véritable «philosophie clinique » de ces années à accompagner des patients jusqu'au bout de leur vie. Ces soignants pourraient partager leur vision avec les nouveaux venus, ils contribueraient alors à dédramatiser le travail en cancérologie et peut-être les non-dits des patients face à la maladie.

\section{Faire bouger les idées reçues}

Les représentations de la maladie cancer sont encore chargées d'idées reçues qui méritent une réflexion théorique et pratique dans tous les services médicaux, mais aussi dans la société. Leur place est incontestable lorsqu'elles permettent un travail symbolique et même symboligène chez malades et soignants. Les études présentées ici attestent cependant de leur puissance néfaste parfois. C'est donc à nous tous de limiter leurs effets paralysants. Les nier serait sans conséquence. Les mettre au grand jour et les discuter semble plus efficace, les groupes de malades et de soignants le montrent, les témoignages existentiels des uns et des autres aussi.

\section{Références}

1. Bacqué MF, Baillet F (2009) La force du lien face au cancer. Paris, Odile Jacob

2. Freud F (1914, nouvelle édition 1988) Métapsychologie. In : Oeuvres Complètes, Paris, PUF 